\title{
COMPONENTS OF AN OPEN SET
}

\author{
MARK MANDELKERN \\ (Received 17 August 1981; revised 26 October 1981) \\ Communicated by J. H. Rubinstein
}

\begin{abstract}
A classical theorem states that any open set on the real line is a countable union of disjoint open intervals. Here the numerical content of this theorem is investigated with the methods of constructive topology.
\end{abstract}

1980 Mathematics subject classification (Amer. Math. Soc.): 26 A 03, 54 A 99.

\section{Introduction}

A complete structural characterization of an open set on the real line is given by its resolution into a countable union of disjoint open intervals. The resolution is also useful in understanding the structure of closed sets: a closed set is formed by removing from the line a sequence of disjoint open intervals. The construction of the Cantor set by the removal of the middle thirds is a well-known example.

However, the usual proofs of this theorem are unsatisfactory on several grounds. In the first place, they provide no explicit procedure for finding the component of a given point, that is, for calculating the endpoints of the open interval. Secondly, the usual proofs fail to give an explicit procedure for listing the countably many components in a simple sequence.

Furthermore, examples below will show that the theorem in question is in fact not true for an arbitrary open set, when interpreted in the strong sense of the existence of such explicit procedures.

In the case of bounded sets, the first deficiency in the older proofs has been removed in [5], which gives a procedure for calculating the endpoints of the

(C) Copyright Australian Mathematical Society 1982 
components. This procedure applies not to all open sets, but to the most important of them, the colocated sets (which are defined below).

In this paper, the second deficiency is removed; the components of a bounded colocated set are explicitly arranged in a simple sequence. Applications are given to problems in connectivity and convexity. A characterization of colocated sets is given which shows their special position among the open sets. Counterexamples are given which show the need for the special hypotheses used in the various results and applications.

Only constructive properties of the real line, such as are found in the work of Bishop [1], will be admitted. For example, in lieu of the classical trichotomy, which is nonconstructive, we frequently use the constructively valid property that if $a<b$, then for any point $x$, either $x<b$ or $x>a$.

\section{Components of a bounded colocated set}

The concept of a located set is due to Brouwer: a set $G$ in a metric space $X$ is located if the distance $\rho(x, G)$ for any point $x$ in $X$ can be calculated in a constructive manner. The metric complement $-G$ of a located set $G$ consists of all points at a positive distance from $G$. A set is colocated if it is the metric complement of some located set. A family of sets is said to be disjoint if any two which have a common point are equal.

In Theorem 4 of [5], a bounded colocated set $U$ on the line was shown to be a union of disjoint open intervals, of the form $U=\cup_{a \in U} I_{a}$, where the index set is $U$ itself, and the component of any point $a$ in $U$ is the interval $I_{a}$ with constructively defined endpoints.

THEOREM 1. Any bounded colocated set on the line is a countable union of disjoint open intervals.

Proof. Let $U=\cup_{a \in U} I_{a}$ be the resolution of the bounded colocated set $U$ into components as obtained in [5], and let $G$ be a located set with $U=-G$. Enumerate the set $\left\{(q, k): q \in Q, k \in \mathbf{Z}^{+}\right\}$as a simple sequence $\left\{\left(q_{n}, k_{n}\right)\right\}_{n=1}^{\infty}$. Construct a sequence $\left\{\sigma_{n}\right\}$ of 0 's and l's such that $\rho\left(q_{n}, G\right)<1 / k_{n}$ when $\sigma_{n}=0$, and $\rho\left(q_{n}, G\right)>0$ when $\sigma_{n}=1$. For each $n$, define $J_{n} \equiv \varnothing$ if $\sigma_{n}=0$, and $J_{n} \equiv I_{q_{n}}$ when $\sigma_{n}=1$, where $I_{q_{n}}$ is the component of $q_{n}$ in the above resolution. This defines a countable family of disjoint open intervals whose union $\cup_{n=1}^{\infty} J_{n}$ is contained in $U$. Now let $x \in U$. Construct $q$ in $I_{x}$, choose $k$ so that $1 / k<\rho(q, G)$, and choose $n$ so that $\left(q_{n}, k_{n}\right)=(q, k)$. It follows that $\sigma_{n}=1$. Since $q \in I_{x}$, then $x \in I_{q}=J_{n}$. Hence $U=\cup_{n=1}^{\infty} J_{n}$. 
The following lemma shows that the components of a bounded colocated set satisfy a stronger disjointness condition.

LEMMA 1. If a bounded open set on the line has any resolution into disjoint open intervals, then any two nonvoid components are either equal or disjoint.

Proof. Let $I_{\alpha}$ and $I_{\beta}$ be nonvoid components in a resolution $\cup_{\alpha \in A} I_{\alpha}$, and let $I_{\alpha}=(a-r, a+r)$ and $I_{\beta}=(b-s, b+s)$, where $r>0$ and $s>0$. In the case $|a-b|<r$, then $b \in I_{\alpha}$ and it follows that $I_{\alpha}=I_{\beta}$. Now consider the case $|a-b|>0$, and suppose $I_{\alpha}$ and $I_{\beta}$ have a common point. Then these components are equal, and $a=b$, which is a contradiction. Hence in this case the components are disjoint.

The following lemma is immediate.

Lemma 2. The components of an open set $U$ on the line, when they exist, are unique. If $U=\cup_{\alpha \in A} I_{\alpha}$ and $U=\cup_{\beta \in B} J_{\beta}$ are two resolutions of $U$ into unions of disjoint open intervals, then for any nonvoid component $I_{\alpha}$ there exists a component $J_{\beta}$ such that $I_{\alpha}=J_{\beta}$.

The following corollary shows that the components of a colocated set may be constructed so as to satisfy a disjointness condition stronger yet than that of Lemma 1.

Corollary 1. Any bounded colocated set on the line is a union $\cup_{k=1}^{\infty} I_{k}$ of open intervals such that

(1) $I_{j}$ and $I_{k}$ are disjoint whenever $j \neq k$, and

(2) each interval $I_{k}$ is either void or nonvoid.

Proof. The construction given for Theorem 1 yields components each of which is either void or nonvoid. Lemma 1 shows that a given component is either disjoint from all preceding components or equal to one of them, in which case it may be replaced by the void interval.

A set $E$ is countable if there exists a surjection $f: \mathbf{Z}^{+} \rightarrow E$, that is, if the elements of $E$ can be arranged in a sequence with possible duplications (see page 15 in [1]). A subset $T$ of a set $S$ is a free subset if for any point $x$ in $S$, either $x \in T$, or $x \in T$ is contradictory (see page 65 in [1]). Although nonvoid free subsets of a countable set are countable, in Section 8 it will be shown that not 
every nonvoid subset of a countable set is countable. Thus a countable resolution cannot be obtained from the resolution $U=\cup_{a \in U} I_{a}$, by merely writing $U=$ $\bigcup_{q \in U \cap Q} I_{q}$.

A subset $T$ of a set $S$ will be called sequentially free if for any point $x$ in $S$ there exists a nondecreasing sequence $\left\{\sigma_{n}\right\}$ of 0 's and l's such that $x$ is in $T$ if and only if some $\sigma_{n}=1$. Although not every colocated set in a metric space $X$ is free, every colocated set $U=-G$ is sequentially free. (To see this, note that for any point $x$ in $X$, and any positive integer $n$, either $\rho(x, G)<1 / n$ or $\rho(x, G)>0$. Thus we may define $\sigma_{n} \equiv 0$ or $\sigma_{n} \equiv 1$ accordingly.) Similarly, every closed located set $F$ is consequentially free, that is, for any point $x$ in $X$ there exists a nondecreasing sequence $\left\{\sigma_{n}\right\}$ of 0 's and l's such that $x$ is in $F$ if and only if all $\sigma_{n}=0$.

The method used in the proof of Theorem 1 may be applied more generally as follows.

Proposition 1. If $T$ is a sequentially free subset of a set $S$, if $E$ is a countable subset of $S$, and if $T$ meets $E$, then $T \cap E$ is countable. Hence every nonvoid sequentially free subset of a countable set is also countable.

Proof. Let $E \equiv\left\{x_{1}, x_{2}, \ldots\right\}$ be an enumeration of $E$; we may assume $x_{1} \in T$. For each $i$, construct a nondecreasing sequence $\left\{\sigma_{n}^{i}\right\}_{n}$ of 0 's and l's so that $x_{i} \in T$ if and only if $\sigma_{n}^{i}=1$ for some $n$. For all $i$ and all $n$, define $y_{n}^{i} \equiv x_{1}$ if $\sigma_{n}^{i}=0$, and $y_{n}^{i} \equiv x_{i}$ if $\boldsymbol{\sigma}_{n}^{i}=1$. It follows that $T \cap E=\left\{y_{n}^{i}: i, n=1,2, \ldots\right\}$, which is a countable set.

It follows that the rational points of any nonvoid colocated set on the line may be enumerated.

\section{Applications in connectivity}

In Theorem 1 of [5] it was shown that two disjoint nonvoid open subsets of a bounded interval cannot cover the interval, provided both subsets are located. In [3] it is shown that it suffices to assume that one of the subsets is located; an example in section 9 below shows that not all auxiliary conditions can be removed. The result will now be obtained under the condition that one of the subsets is colocated.

Let $S$ be a set with an inequality relation. If $x$ is an element of $S$ and $A$ is a subset of $S$, write $x \notin A$ if $x \neq y$ for all $y$ in $A$. In addition, define $A^{*} \equiv\{x \in S$ : $x \notin A\}$. In a metric space, $x \neq y$ means $\rho(x, y)>0$, and thus $x \notin A$ means 
$\rho(x, y)>0$ for all $y$ in $A$. An open set $U$ in a metric space has a special property: $x \notin U$ if and only if $x \in U$ is contradictory.

COROLlary 2. Let $U$ and $V$ be disjoint nonvoid open subsets of a bounded interval $I$, one of which is colocated. Then there exists a point $x$ in $I$ with $x \notin U \cup V$.

Proof. Let $U$ be colocated, construct a nonvoid component $(a-r, a+r)$ of $U$, and construct an element $b$ in $V$. We may assume $a<b$; it follows that $x \equiv a+r$ is a point of $I$ such that $x \notin U$ and $x \notin V$.

The next two results are related to results on connectivity in [2] and [4]; in lieu of the located sets considered there, these concern colocated sets.

COROLlaRY 3. Let $U$ be a nonvoid colocated subset of the open unit interval $I \equiv(0,1)$. If $U$ is closed in $I$, then $U=I$.

Proof. Let $(a, b)$ be a nonvoid component of $U$; then $b \notin U$. If $b<1$ then $b$ is in the closure of $U$ in $I$, a contradiction; hence $b=1$. Similarly, $a=0$.

COROLlaRY 4. Let $U$ be a nonvoid colocated subset of the open unit interval $I \equiv(0,1)$. If $U$ is (in a strong sense) not all of $I$, then $U$ is (in a strong sense) not closed in $I$. That is, if there exists a point $y$ in I with $y \notin U$, then there exists a point $z$ which lies in the closure of $U$ in $I$, and with $z \notin U$.

Proof. Let $(a, b)$ be a nonvoid component of $U$. Since $y \notin U$, it follows that either $y \leqslant a$ or $y \geqslant b$. We may assume $y \leqslant a$, and define $z \equiv a$.

\section{An application in convexity}

COROLLARY 5. Every nonvoid bounded colocated convex set on the line is an open interval.

Proof. In the resolution given by Corollary 1 , one component must be nonvoid, and the others void. Alternatively, the lemma in [5] may be applied directly.

Examples 6 and 7 in Section 10 will show that the conditions "colocated" and "nonvoid" can not be removed. 


\section{Characterization of colocated sets}

Since the main results above are obtained only for colocated sets, in lieu of arbitrary open sets, colocated sets will now be characterized among the open sets. For a subset $A$ of a metric space $X$, we utilize the set $A^{*}$ defined in Section 3; thus $A^{*}=\{x \in X: \rho(x, y)>0$ for all $y \in A\}$. It is easily seen that when $U$ is open, then $U^{*}$ is closed.

THEOREM 2. A subset $U$ of a metric space $X$ is colocated if and only if

(1) $U$ is open,

(2) $U^{*}$ is located, and

(3) for any $x$ in $X$ and any $\delta>0$, either $x$ lies in $U$, or some point of the open sphere $S(x, \delta)$ lies in $U^{*}$.

In this situation, $U=-U^{*}$.

Proof. First let $U$ be colocated; thus there exists a located set $G$ with $U=-G$. It is easily seen that $U^{*}$ is located with $\rho\left(x, U^{*}\right)=\rho(x, G)$ for all $x$ in $X$. Also, condition (3) is immediate.

Now let $U$ satisfy the three conditions. Since $U$ is open, it follows that $U \subset-U^{*}$. Let $x \in-U^{*}$; then $\delta \equiv \rho\left(x, U^{*}\right)>0$. Any point of $S(x, \delta)$ also lies in $-U^{*}$; thus it follows from condition (3) that $x$ is in $U$. Hence $U=-U^{*}$ and thus $U$ is colocated.

An example will be given in Section 12 to show that condition (3) may not be removed.

Corollary 6. A colocated set $U$ is the metric complement of a unique closed located set, namely $U^{*}$. The family of located sets of which $U$ is the metric complement is the family of dense subsets of $U^{*}$.

\section{Counterexamples-introduction}

The resolution of an open set on the line into a countable union of disjoint open intervals is obtained above only for a bounded colocated set. The examples below will show to what extent these restrictions, and the restrictions used in the applications, are necessary.

Classically (that is, nonconstructively), every open set on the line is a union of disjoint open intervals, but not constructively. The italicized word "not" used 
here has a special meaning in studies of constructivity. It means that a counterexample exists in the sense of Brouwer. Such a counterexample consists of a proof showing that the statement in question implies one of several principles which seems constructively invalid. That is, no proofs of the principles are known, and it seems unlikely that constructive proofs will ever be found. For example, the statement "every bounded open set on the line is a union of disjoint open intervals" implies the limited principle of omniscience (LPO). LPO states that there is a general procedure which applies to any given sequence of integers and determines, by a finite process, either that each term of the sequence is zero or that some (specifically presented) term is nonzero. Further analysis of this principle indicates that such a procedure is not likely to be discovered. Therefore, because of the implication given in the counterexample, it is unlikely that a proof of the above statement in quotes will ever be found. Further discussion of Brouwerian counterexamples is found in Chapters 1 and 2 of [1].

Consider the following succession of four conditions concerning a bounded set $U$ on the line:

(1) $U$ is colocated.

(2) $U$ is a countable union of disjoint open intervals.

(3) $U$ is a union of disjoint open intervals.

(4) $U$ is open; that is, $U$ is a union of open intervals.

Each condition implies the next; examples will show that each converse is not true.

We will also construct an example of an unbounded colocated set that is not a union of disjoint open intervals, and examples concerning connectivity, convexity, and countability. The principle of finite possibility will be introduced and will be used in giving counterexamples in the same way that various versions of the principle of omniscience are used.

\section{Examples with LPO}

The omniscience principles used in the counterexamples are most easily expressed in terms of nondecreasing sequences $\left\{a_{n}\right\}_{n=1}^{\infty}$ of 0 's and l's; such sequences will be called decision sequences.

The limited principle of omniscience (LPO) will be used in the following equivalent form: If $\left\{a_{n}\right\}$ is any decision sequence, then either $a_{n}=0$ for all $n$, or there exists $n$ (through an explicit construction) such that $a_{n}=1$.

We first construct a bounded open set that is not a union of disjoint open intervals. 
EXAmple 1. "Every bounded open set on the line is a union of disjoint open intervals" implies LPO.

Proof. Let $\left\{a_{n}\right\}$ be a decision sequence and define $U \equiv \cup_{n=1}^{\infty}\left(0, a_{n}+1\right)$. By hypothesis, $U$ has a resolution $U=\cup_{\alpha \in A} I_{\alpha}$ into disjoint open intervals. Construct the component $I_{\alpha}=(a, b)$ of the point $1 / 2$ in $U$. In the case $b<2$, it follows that all $a_{n}=0$. In the case $b>1$, the point 1 lies in $U$, and thus $a_{n}=1$ for some $n$.

Although the set $U$ of Example 1 is not in general an interval, in specific instances it may happen that $U=(0,1)$, or it could happen that $U=(0,2)$. Even for a specific decision sequence for which neither alternative is known at the present time, more information obtained in future might result in one of these alternatives. It is this varied possibility, and the lack of any general finite process for determining such definite alternatives, that prevents us from giving a general finite process for determining the component of the point $1 / 2$.

Another interesting set which could have been used in Example 1 is $U \equiv$ $(-1, \alpha) \cup(0,1)$, where $\alpha \geqslant 0$ but it is not known whether $\alpha>0$ or $\alpha=0$; for example, $\alpha \equiv \sum a_{n} / 2^{n}$, where $\left\{a_{n}\right\}$ is an arbitrary decision sequence.

We now construct a countable union of disjoint open intervals that is not colocated.

EXAMPLE 2. "Every bounded countable union of disjoint open intervals is colocated" implies LPO.

Proof. Let $\left\{a_{n}\right\}$ be a decision sequence and define $U \equiv \cup_{n=1}^{\infty}\left(a_{n}, a_{n}+1\right)$. By hypothesis there exists a located set $G$ with $U=-G$. In the case in which $\rho(3 / 2, G)<1 / 2$, construct $x$ in $G$ so that $1<x<2$. It then follows that all $a_{n}=0$. In the case in which $\rho(3 / 2, G)>0$, then the point $3 / 2$ lies in $U$, and thus some $a_{n}=1$.

\section{The principle of finite possibility}

From the succession of four conditions listed above in Section 6, it remains only to construct a union of disjoint open intervals that is not a countable union of disjoint open intervals. Now if $U$ is any union of disjoint open intervals, each point $a$ in $U$ has a component $I_{a}$, and thus $U=\bigcup_{a \in U} I_{a}$. Since each nonvoid component contains a rational, then also $U=U_{q \in U \cap Q} I_{q}$, where the index set is a subset of the countable set $\mathbf{Q}$. Hence we are led to consider subsets of countable 
sets generally, and to construct such a set that is itself not countable. A Brouwerian counterexample will proceed from the hypothesis that a certain set is countable, that is, from the existence of a certain sequence. It is improbable that the existence of such a sequence will imply LPO or one of its weaker versions; thus a new principle, also constructively unlikely, is needed.

To find the appropriate principle, we examine the canonical subset of a countable set that cannot be proved countable. Let $\left\{a_{n}\right\}$ be a decision sequence, and let $T$ be the subset of $\mathbf{Z}^{+}$that contains 0 , and also contains 1 in the event that all $a_{n}=0$. The set $T$ may also be defined in (seemingly) more conventional terms by defining $\alpha \equiv \sum a_{n} / 2^{n+1}$ and $T \equiv\{0,1+\alpha\} \cap \mathbf{Z}^{+}$. Alternatively, define $T \equiv$ $\{0\} \cup\left\{m \in Z^{+}: m=a_{n}+1\right.$ for all $\left.n\right\}$. Now if $T$ were countable there would be an enumeration $T=\left\{t_{1}, t_{2}, \ldots\right\}$, and it would follow that all $a_{n}=0$ if and only if some $t_{n}=1$. It seems unlikely that we will ever find a general procedure for constructing such a sequence $\left\{t_{n}\right\}$ corresponding to any decision sequence $\left\{a_{n}\right\}$.

Principle of Finite Possibility (PFP). For any decision sequence $\left\{a_{n}\right\}$, there exists a corresponding decision sequence $\left\{t_{n}\right\}$ such that all $a_{n}=0$ if and only if some $t_{n}=1$.

The terminology reflects the fact that whereas it is impossible, by merely calculating values of the terms $a_{n}$ in succession, to conclude after only finitely many calculations that all $a_{n}=0$, it is conceivable that this determination might be possible after the calculation of only finitely many of the terms $t_{n}$. An equivalent form of PFP is: For every real number $\alpha \geqslant 0$ there exists a real number $\beta \geqslant 0$ such that $\alpha=0$ if and only if $\beta>0$.

EXAMPLE 3. "Every nonvoid subset of a countable set is also countable" implies PFP.

Proof. Given a decision sequence $\left\{a_{n}\right\}$, the set $T$ above provides the sequence $\left\{t_{n}\right\}$.

Thus we have constructed a nonvoid subset of a countable set that is not countable, where "not" is now also used to refer to counterexamples with PFP. For example, a counterexample with PFP shows that $\mathbf{Q}$ is not sequentially free; let $\left\{a_{n}\right\}$ be a decision sequence, defined $\alpha=\sum a_{n} / 2^{n}$, and consider the point $x \equiv \alpha \sqrt{2}$.

An equivalent form of PFP is given by the following.

Proposition 2. "If $F$ is a closed located set on the line, and $E$ is a countable set on the line that meets $F$, then $F \cap E$ is countable" is equivalent to PFP. 
Proof. To show that the given statement implies PFP, let $\left\{a_{n}\right\}$ be a decision sequence, define $\alpha \equiv \Sigma a_{n} / 2^{n+1}$, define $F \equiv\{0,1+\alpha\}$, and let $E$ be the set of integers. Then $F \cap E$ is the set $T$ constructed above. Thus PFP obtains.

Conversely, under PFP, a closed located set is sequentially free, and Proposition 1 applies.

We now construct a set that is a union of disjoint open intervals, but not a countable union of disjoint open intervals.

EXAMPLE 4. "Every bounded union of disjoint open intervals is a countable union of disjoint open intervals" implies PFP.

Proof. Let $\left\{a_{n}\right\}$ be a decision sequence and define $T$ as above. Define $U \equiv \cup_{t \in T}(t, t+1)$; thus $U$ is a union of disjoint open intervals. By hypothesis, $U$ has a countable resolution $U=\cup_{n=1}^{\infty} I_{n}$ into disjoint open intervals. Consider any component $I_{n}=(a, b)$ and apply Lemma 2 . In the case that $b-a<1$ it follows that $I_{n}$ is void. In the case that $b-a>0$, then either $I_{n}=(0,1)$ or $I_{n}=(1,2)$. Construct a decision sequence $\left\{t_{n}\right\}$ so that when $t_{n}=0$, then $I_{n}=\varnothing$ or $I_{n}=(0,1)$, and when $t_{n}=1$, then $I_{k}=(1,2)$ for some $k \leqslant n$. It follows that all $a_{n}=0$ if and only if some $t_{n}=1$.

To relate PFP to other omniscience principles, let $\left\{a_{n}\right\}$ be a decision sequence, assume PFP, and construct the corresponding decision sequence $\left\{t_{n}\right\}$. Following a suggestion of Errett Bishop, we construct a decision sequence $\left\{s_{n}\right\}$ by defining $s_{n} \equiv a_{n} \vee t_{n}$. Now assume the limited principle of existence (LPE): If $\left\{b_{n}\right\}$ is a decision sequence such that it is contradictory that all $b_{n}=0$, then some $b_{n}=1$. (An equivalent form of LPE is: If $x$ is a real number and it is contradictory that $x \leqslant 0$, then $x>0$.) Now suppose all $s_{n}=0$; then all $a_{n}=0$, and it follows that some $t_{n}=1$, which is a contradiction. By LPE, we construct an integer $k$ so that $s_{k}=1$. Thus either $t_{k}=1$ or $a_{k}=1$, and LPO follows.

Hence LPO is equivalent to PFP and LPE combined. Since it is very unlikely that LPE will ever be shown equivalent to LPO, this contributes to the unlikelihood of PFP.

\section{An example in connectivity}

Here we construct disjoint nonvoid open subsets $U$ and $V$ of a bounded interval for which there does not exist a point $x$ in the interval such that $x \notin U \cup V$ (see Corollary 2). The example uses the weak limited principle of 
omniscience (WLPO): If $\left\{a_{n}\right\}$ is any decision sequence, then either all $a_{n}=0$ or it is contradictory that all $a_{n}=0$.

EXAMPLE 5. "Whenever two nonvoid open subsets $U$ and $V$ of a bounded interval are disjoint, there exists a point $x$ in the interval such that $x \notin U \cup V$ " implies WLPO.

Proof. Let $\left\{a_{n}\right\}$ be a decision sequence and define $A=\left\{a_{n}: n \in \mathbf{Z}^{+}\right\}$. Let $T$ be the set of Section 8, so that $0 \in T$, and $1 \in T$ if and only if all $a_{n}=0$. Define $U \equiv \cup_{a \in A}(0,1+a)$ and $V \equiv \cup_{t \in T}(2-t, 3)$. If $U$ and $V$ meet, there exist points $a$ in $A$ and $t$ in $T$ such that $2-t<1+a$, and it follows that $a=t=1$, which is contradictory. Hence $U$ and $V$ are disjoint. By hypothesis, there exists a point $x$ in $(0,3)$ with $x \notin U \cup V$. In the case $x<2$, it follows that all $a_{n}=0$. In the case $x>1$, then it is contradictory that all $a_{n}=0$.

\section{Examples in convexity}

Here the counterexamples relating to Corollary 5 will be constructed. The set already constructed in Example 1 is a nonvoid bounded open convex set that is not an interval, and thus provides the following counterexample.

EXAmple 6. "Every nonvoid bounded open convex set on the line is an open interval" implies LPO.

We now construct a bounded colocated convex set that is not an interval. The example uses the lesser limited principle of omniscience (LLPO): If $\left\{a_{n}\right\}$ is any decision sequence, then either "if some $a_{n}=1$ then the least such integer $n$ is even", or "if some $a_{n}=1$ then the least such integer $n$ is odd".

EXAMPLE 7. "Every bounded colocated convex set on the line is an open interval" implies LLPO.

Proof. Let $\left\{a_{n}\right\}$ be a decision sequence. In the event that some $a_{n}=1$, define $k$ to be the first such integer $n$. For any $n$, if $a_{n}=0$, define $G_{n} \equiv[1 / n, 1-1 / n]$, while if $a_{n}=1$, define $G_{n} \equiv[0,1-1 / k]$ when $k$ is even, and $G_{n} \equiv[1 / k, 1]$ when $k$ is odd. Define $G \equiv(-\infty, 0] \cup \cup_{n=1}^{\infty} G_{n} \cup[1,+\infty)$. Given any point $x$ on the line, one may calculate $a_{n}$ for any $n$, and thus one may calculate $\rho(x, G)$ to within $1 / n$. Hence $G$ is located. Define $U \equiv-G$. If any point $x$ of $U$ is given, choose $n$ so that $1 / n<\rho(x, G)$; it follows that $a_{n}=1$. This shows that $U$ is convex. By 
hypothesis, there exist real numbers $a$ and $b$ such that $U=(a, b)$. Either $a>0$, and then $k$ would be even, or $a<1 / 2$, and then $k$ would be odd.

\section{Unbounded colocated sets}

Now consider a colocated set without the restriction that it be bounded. If this set has a resolution into disjoint open intervals, then since a component may be infinite, extended real numbers may be required to specify the endpoints. An extended real number is either a real number or $+\infty$ or $-\infty$ (see page 33 in [1]). Thus an open interval is either bounded or unbounded. For this reason a colocated set need not be a union of disjoint open intervals.

EXAMPLE 8. "Every colocated set on the line is a union of disjoint open intervals" implies LPO.

Proof. Let $\left\{a_{n}\right\}$ be a decision sequence; we may assume $a_{1}=0$. Define $G \equiv\{0, k\}$, where $k$ is the least integer $n$ such that $a_{n}=1$, if any such integers exist. That is, $G \equiv\{0\} \cup\left\{n \in Z^{+}: a_{n-1}<a_{n}\right\}$. Given a point $x$ on the line, choose $n>2|x|$ and calculate $a_{n}$. If $a_{n}=0$, then $\rho(x, G)=|x|$; while if $a_{n}=1$, then $\rho(x, G)=|x| \wedge|x-k|$, where $a_{k-1}<a_{k}$. Hence $G$ is located, and $U \equiv-G$ is colocated. By hypothesis, $U$ has a resolution $U=\cup_{\alpha \in A} I_{\alpha}$ into disjoint open intervals. Construct the component $I_{\alpha}=(a, b)$ of the point 1 in $U$. In the case in which $b$ is finite, it follows that $b$ is a point of $G$ with $b>0$, and thus some $a_{n}=1$. In the case in which $b= \pm \infty$, it follows that all $a_{n}=0$.

\section{An example on the charcterization of colocated sets}

Here we construct an open set $U$ so that $U^{*}$ is located, but $U$ is not colocated (see Theorem 2).

EXAmple 9. "If $U$ is a nonvoid open set on the line such that $U^{*}$ is located, then $U$ is colocated" implies LPO.

Proof. Let $A$ and $T$ be as in Example 5. Define $U_{1} \equiv \cup_{a \in A}(0, a)$, and $U_{2} \equiv \cup_{t \in T}(0, t)$, and $U \equiv U_{1} \cup U_{2} \cup(1 / 2,1)$. Suppose there is a point $x$ in $(0,1) \cap U^{*}$. Then $x \notin U_{1}$ and hence all $a_{n}=0$; it follows that $1 \in T$ and thus 
$x \in U_{2}$, a contradiction. Thus $(0,1) \cap U^{*}$ is void. It follows that $U^{*}=(-\infty, 0]$ $\cup[1,+\infty)$; and thus $U^{*}$ is located. By hypothesis, $U$ is colocated; it follows from Theorem 2 that $U=-U^{*}=(0,1)$. Thus the point $1 / 4$ lies in $U$. It follows that either $1 / 4 \in U_{1}$, and hence some $a_{n}=1$, or $1 / 4 \in U_{2}$, and hence all $a_{n}=0$.

\section{References}

[1] E. Bishop, Foundations of constructive analysis (McGraw-Hill, New York, 1967).

[2] D. S. Bridges, 'On the connectivity of convex sets', Bull. London Math. Soc. 10 (1978), 86-90.

[3] D. S. Bridges, 'More on the connectivity of convex sets', Proc. Amer. Math. Soc. 68 (1978), 214-216.

[4] D. S. Bridges, 'Connectivity properties of metric spaces', Pacific J. Math. 80 (1979), 325-331.

[5] M. Mandelkern, 'Connectivity of an interval,' Proc. Amer. Math. Soc. 54 (1976), 170-172.

\section{Department of Mathematical Sciences}

New Mexico State University

Las Cruces, New Mexico 88003

U.S.A. 